\title{
Article \\ Concordance of Alzheimer's Disease Subtypes Produced from Different Representative Morphological Measures: A Comparative Study
}

\author{
Baiwen Zhang ${ }^{1,2}$, , Lan Lin ${ }^{1, *}$, Lingyu Liu ${ }^{1}$, Xiaoqi Shen ${ }^{1}$ and Shuicai $\mathrm{Wu}^{2, *}$ \\ 1 Department of Biomedical Engineering, Faculty of Environment and Life Sciences, \\ Beijing University of Technology, Beijing 100124, China; zhangby@emails.bjut.edu.cn (B.Z.); \\ llyy123llyy@sina.com (L.L.); xiaoqishen@emails.bjut.edu.cn (X.S.) \\ 2 Intelligent Physiological Measurement and Clinical Translation, Beijing International Base for Scientific and \\ Technological Cooperation, Beijing University of Technology, Beijing 100124, China \\ * Correspondence: lanlin@bjut.edu.cn (L.L.); wushuicai@bjut.edu.cn (S.W.); Tel.: +86-10-6739-1610 (L.L. \& S.W.)
}

Citation: Zhang, B.; Lin, L.; Liu, L.; Shen, X.; Wu, S. Concordance of Alzheimer's Disease Subtypes Produced from Different Representative Morphological Measures: A Comparative Study. Brain Sci. 2022, 12, 187. https:// doi.org/10.3390/brainsci12020187

Academic Editor: Toshikazu Ikuta

Received: 4 January 2022

Accepted: 25 January 2022

Published: 30 January 2022

Publisher's Note: MDPI stays neutral with regard to jurisdictional claims in published maps and institutional affiliations.

Copyright: (C) 2022 by the authors. Licensee MDPI, Basel, Switzerland. This article is an open access article distributed under the terms and conditions of the Creative Commons Attribution (CC BY) license (https:// creativecommons.org/licenses/by/ $4.0 /)$.

\begin{abstract}
Background: Gray matter (GM) density and cortical thickness (CT) obtained from structural magnetic resonance imaging are representative GM morphological measures that have been commonly used in Alzheimer's disease (AD) subtype research. However, how the two measures affect the definition of AD subtypes remains unclear. Methods: A total of 180 AD patients from the ADNI database were used to identify AD subgroups. The subtypes were identified via a data-driven strategy based on the density features and CT features, respectively. Then, the similarity between the two features in AD subtype definition was analyzed. Results: Four distinct subtypes were discovered by both density and CT features: diffuse atrophy AD, minimal atrophy AD (MAD), left temporal dominant atrophy AD (LTAD), and occipital sparing AD. The matched subtypes exhibited relatively high similarity in atrophy patterns and neuropsychological and neuropathological characteristics. They differed only in MAD and LTAD regarding the carrying of apolipoprotein E $\varepsilon 2$. Conclusions: The results verified that different representative morphological GM measurement methods could produce similar AD subtypes. Meanwhile, the influences of apolipoprotein E genotype, asymmetric disease progression, and their interactions should be considered and included in the AD subtype definition. This study provides a valuable reference for selecting features in future studies of AD subtypes.
\end{abstract}

Keywords: Alzheimer's disease; gray matter density; cortical thickness; structural magnetic resonance imaging; matched Alzheimer's disease subtypes

\section{Introduction}

It is generally accepted that the neurofibrillary tangles (NFTs) of Alzheimer's disease (AD) derive from the entorhinal cortex, then spread subsequently to the association cortex via the hippocampus, and finally invade the primary cortex [1]. However, several recent studies have suggested that AD patients have striking differences in neuropathologic distinctions, cognitive functions, demographics, and clinical progression, indicating that $\mathrm{AD}$ is a heterogeneous disease with different subtypes [2,3]. The definition of the AD subtype is an essential part of capturing the heterogeneity. As proved by some studies, the gray matter (GM) change in brain region is closely associated with tau pathology in AD patients with abnormal amyloids [4]. Therefore, GM atrophy pattern-based morphometric measures have received increasing attention in the studies of biologically defined AD subtypes [5,6].

Given the high-dimensionality of structural magnetic resonance imaging (sMRI), automated region-based analyses have been conducted in the studies of AD subtypes by parcellating the brain into anatomically defined regions. GM density and cortical thickness (CT) obtained from sMRI are two widely used morphometric features. Both GM features 
respectively constitute multimode features with pathological information, so they have been applied to studies on AD subtypes [7,8]. The GM density for each region-of-interest (ROI) is computed as the sum of GM densities of all voxels within the ROI from the voxel-based morphometry (VBM) maps using statistical parametric mapping (SPM). The surface-based CT value is obtained by averaging the vertex CT values within all FreeSurfer cortical ROIs $[9,10]$. The study results may be influenced by the technical differences in VBM and FreeSurfer, both of which rely on ROIs parcellation that uses a predefined template to identify morphometric changes, but defined ROIs are quite different. CT is one of the hallmark features of AD, and a greater degree of cortical thinning in a disease-affected region indicates the higher severity of AD [11]. However, CT analysis fails to detect the abnormalities in subcortical areas such as the hippocampus [12]. Furthermore, in the early stage of cognitive decline, CT analysis may be relatively insensitive to the abnormalities of some medial temporal lobe (MTL) regions [13]. The density-based approach provides complementary information to the surface-based analysis, thus effectively probing the atrophied subcortical structures [14]. In several density-based studies, the MTL AD subtype experiences severe atrophy in parts of the limbic system such as the hippocampus and other subcortical ROIs [15-17], which is also verified by the higher distribution of tau in the hippocampus in Murray's study [2].

These two morphological measures may be correlated to some extent, where CT measures cortical thinning, and the change in GM density measures a mixed change in local CT, cortical folding, and gyrification. It has been suggested in some studies that CT can drive facilitate the change in GM density [18]. However, most previous studies on AD subtypes definition have focused on only one morphological feature. In our earlier study, four AD subtypes were successfully defined based on the CT features [19], among which each subtype presented its own neuropsychological and neuropathological characteristics. Although several distinct AD subtypes have been identified in most studies, they differ in the definition of AD subtypes, which may be partly attributed to different representative morphological measures [3,19]. This raises a crucial question: How do different GM measurement methods affect the definition of AD subtypes? To answer this question, in the present study, AD subtypes were defined by density-based and CTbased features, respectively, for AD patients from the Alzheimer's disease Neuroimaging Initiative (ADNI) database. If the matched subtypes could be generated by different measurement methods, the characteristics of matched subtypes will be further explored. To our knowledge, this study takes the lead in comparing the two GM morphological measures in AD subtype definition.

\section{Materials and Methods}

\subsection{Subjects and MRI Processing}

The ADNI project was launched in 2004 [20]. The first phase (ADNI-1) lasted from 2004 to 2010, during which time the plan was to recruit a total of 800 individuals aged 50-90, including AD: 200, cognitive normal (CN): 200, and mild cognitive impairment (MCI): 400. The information of all participants of this project was derived from ADNI-1 baseline scans (adni.loni.usc.edu). The T1-weighted images were acquired with a $1.5 \mathrm{~T}$ MRI scanner. The scanning parameters were as follows: $\mathrm{TR}=3000 \mathrm{~ms}$, $\mathrm{TE}=3.55 \mathrm{~ms}$, slice thickness $=1.2 \mathrm{~mm}$, voxel size $=1.2 \times 0.94 \times 0.94 \mathrm{~mm}^{3}[21]$.

The density features used were acquired from 'uaspmvbm.csv', downloaded from the ADNI website (https:/ /ida.loni.usc.edu/, accessed on 3 January 2022), which were extracted from VBM analysis via SPM software (version 5). A total of 116 anatomical ROIs (90 in cerebrum and 26 in cerebellum) were defined using the automated anatomical atlas (AAL) [14]. The CT features extracted by FreeSurfer version 4.3 (http:/ / freesurfer.net/, accessed on 3 January 2022) were acquired from ' ucsffx.csv', downloaded from the ADNI website (https: / / ida.loni.usc.edu/, accessed on 3 January 2022). The thickness values are based on the Desikan-Killiany atlas, including 34 parcels per hemisphere [10]. 
Only the subjects having both CT and density features were kept in the study. Fourteen subjects were excluded due to inconsistencies. The screening process of subjects is shown in Figure 1.

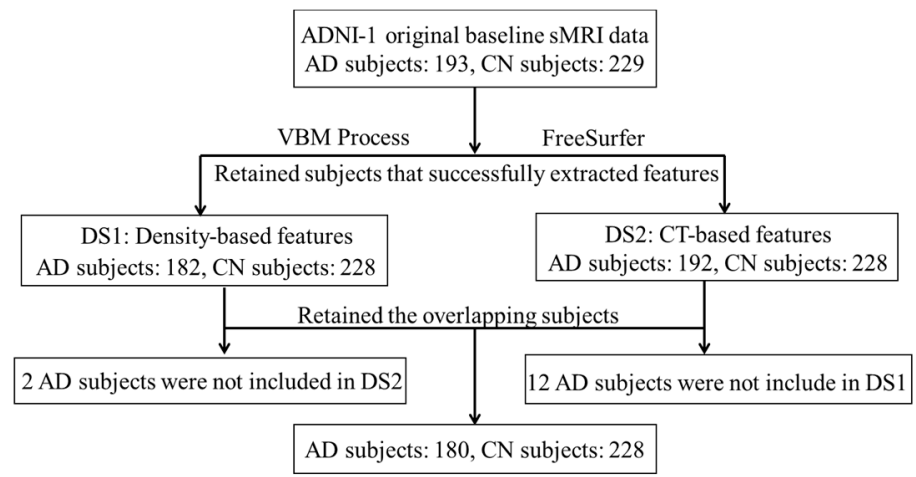

Figure 1. Data screening process. Abbreviations: DS: Data set, ADNI: Alzheimer's disease Neuroimaging Initiative, CT: Cortical thickness, VBM: voxel-based morphometry, AD: Alzheimer's disease, CN: cognitive normal subjects, sMRI: structural MRI.

\subsection{Definition of $A D$ Subtypes}

The features extracted from the screened subjects were input into the mixture of experts (MOE) model to define subtypes [22]. The process of subtypes definition and subsequent analysis is shown in Figure 2.

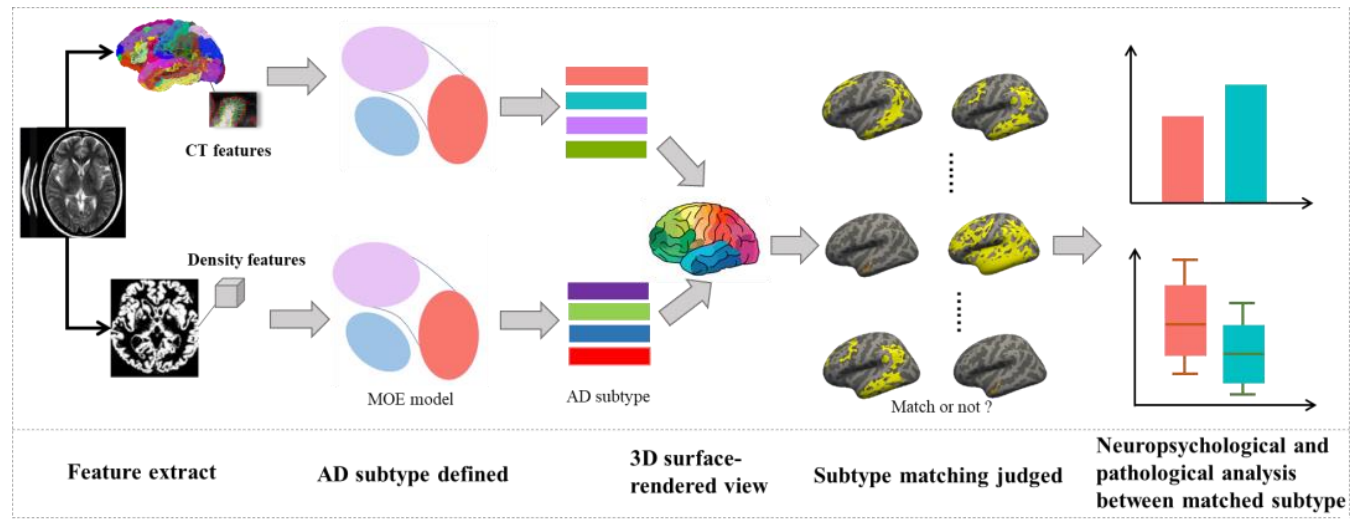

Figure 2. Overview of the steps for AD subtype definition and analysis. Abbreviations: AD: Alzheimer' disease, MOE: mixture of experts model, CT: cortical thickness.

Before the features were input into the MOE model, the age, sex, years of education, and intracranial volume (ICV) were entered as nuisance variables, so they were regressed from the original features. First, the regression coefficient of $\mathrm{CN}\left(\beta_{C N}\right)$ was calculated using a generalized linear model as Formula (1):

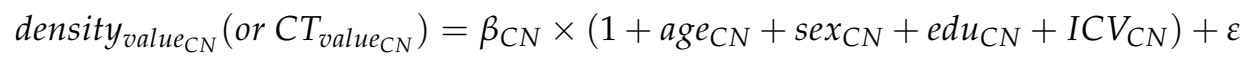

Then, the effects of each nuisance variable were regressed out of all subjects, as Formula (2):

$$
\text { Residual } \left._{\text {All }}=\text { density_value all }_{\left(\text {or } C T \_v a l u e_{a l l}\right.}\right)-\beta_{\text {all }} \times\left(1+a_{g e} e_{\text {all }}+\operatorname{sex}_{\text {all }}+e d u_{\text {all }}+I C V_{\text {all }}\right)
$$

After the regression analysis, each subject was given a binary label, $y_{i} \in\{-1,1\}$. The $\mathrm{CN}$ subjects were set as the reference group $(y=-1)$ and the AD patients as the affected group $(y=1)$. Ten-fold cross-validation was adopted for the MOE. The MOE combined the 
fuzzy c-means (FCM) and support vector machines (SVM). The joint optimization model formula was as follows (3):

$$
\begin{array}{r}
\operatorname{minimize}_{\left\{w^{k}\right\}_{k}\left\{m_{i}^{k}\right\}_{i, k}} \sum_{k=1}^{K}\left\{\frac{1}{2}\left\|w^{k}\right\|_{1}+C \sum_{i=1}^{N} m_{i}^{k}\left(1-y_{i}\left(w^{k}\right)^{T} x_{i}\right)_{+}^{2}+t \sum_{i=1}^{N}\left(m_{i}^{k}\right)^{2}\left\|x_{i}-d^{k}\right\|_{F}^{2}\right\} \\
\text { subject to }: \sum_{k=1}^{K} m_{i}^{k}=1, m_{i}^{k} \in[0,1] \quad n=1, \cdots, N, d^{k}=\frac{\sum_{i=1}^{N}\left(m_{i}^{k}\right)^{\alpha} x^{i}}{\sum_{i=1}^{N}\left(m_{i}^{k}\right)^{\alpha}}
\end{array}
$$

where $K$ is the number of experts, $m$ is the value of membership, $N$ is the number of AD subjects, $C$ is the loss penalty, and $t$ is the trade-off between the cost of SVMs and FCM.

The number of experts was fixed at four. The parameters $C$ and $t$ were simultaneously optimized through the grid search, with a search range of $\left\{2^{-3} \sim 2^{10}\right\}$, respectively. The selection of parameters mainly depended on the cross-validated accuracy (ACC), maximum pair-wise inner-product $\left(\mathrm{W}_{\mathrm{r}}\right)$, and Bezdek partition coefficient (BPC) [23]. Given all those assessments, the density-based AD subtypes and CT-based AD subtypes were defined, respectively. Each subtype was then named according to its atrophic regions.

\subsection{Statistical Analysis}

The characteristics included three main parts: (1) demographic, including age, gender, years of education, onset age, and duration of AD. (2) Cognitive characteristics, including the Mini-Mental State Exam (MMSE), Clinical Dementia Rating Scale-Sum of Boxes (CDRSB), AD Assessment Scale-Cognitive Subscale (ADAS-Cog), and for subdomains ADNI composite scores: memory (ADNI-MEM), executive (ADNI-EF), language (ADNILAN), and visuospatial abilities (ADNI-VS) [24,25]. (3) Apolipoprotein E (APOE) genotypes and cerebrospinal fluid (CSF) biomarker abnormality levels, including APOE $\varepsilon 2, \operatorname{APOE} \varepsilon 4$, beta-amyloid 1-42 (A $\left.\beta_{1-42}\right)$, Phosphorylated tau (P-tau), and Total tau (T-tau). The cut-off value of $\mathrm{A} \beta_{1-42}$, T-tau, and P-tau were $192 \mathrm{ng} / \mathrm{L}, 93 \mathrm{ng} / \mathrm{L}$, and $23 \mathrm{ng} / \mathrm{L}$, respectively [26,27]. The differences between matched subtype were compared. The differences of qualitative variables were calculated via the chi-square tests. The quantitative variables were assessed using analysis of variance (ANOVA), and pairwise comparison by the Dunnett's test. Statistical analysis was performed with SPSS, version 19.0, Armonk, NY, USA.

\section{Results}

\subsection{Subtypes Definition and Matching}

All AD subjects were divided into four subtypes. Considering all the evaluation criteria of $\mathrm{MOE}$, the parameter values were reasonably selected. The optimized parameters and evaluation indicators are shown in Table 1.

Table 1. The parameters selection and evaluation results of MOE.

\begin{tabular}{cccccc}
\hline \multirow{2}{*}{ Method } & \multicolumn{3}{c}{ Optimized Parameters } & \multicolumn{3}{c}{ Evaluation Indicators } \\
\cline { 2 - 6 } & $\boldsymbol{t}$ & $\boldsymbol{C}$ & ACC (\%) & BPC & $\mathbf{W}_{\mathbf{r}}$ \\
\hline Density & 2 & $2^{-3}$ & $77.3(4.4)$ & $0.82(0.03)$ & $0.32(0.44)$ \\
CT & $2^{-2}$ & $2^{-3}$ & $83.1(4.8)$ & $0.63(0.02)$ & $0.29(0.07)$ \\
\hline
\end{tabular}

Abbreviations: $t, C$ : the optimized parameters of MOE, ACC: cross-validated accuracy, BPC: Bezdek partition coefficient, $\mathrm{W}_{\mathrm{r}}$ : maximum pair-wise inner-product; data are presented as mean (standard deviation). CT: cortical thickness.

The CT difference between AD subtype and CN were rendered in the same FreeSurfer atlas to compare the differences of the match subtype (Figure 3). As can be seen from Figure 3, the four subtypes defined by two features were one-to-one matched in cortical atrophy regions, and named diffuse atrophy AD (DAD), minimal atrophy AD (MAD), left temporal dominant atrophy AD (LTAD), and occipital sparing AD (OSAD), respectively.

The subjects in the matched subtype overlapped greatly, and their Dice scores, calculated according to formula (4), ranged from $71.8 \%$ to $84.8 \%$. The 'Subjects' in the formula 
were from the matched subtype. The number of overlapping subjects (intersection subjects) and the results of the Dice score in each matched subtype is indicated in Figure 3. The characteristics of each subtype are summarized in Table 2.

$$
\text { Dice score }=\frac{2 \times \mid \text { Subjects }_{\text {density-based }} \cap \text { Subject }_{C T-\text { based }} \mid}{\mid \text { Subjects }_{\text {density-based }}|+| \text { Subject }_{C T-\text { based }} \mid}
$$
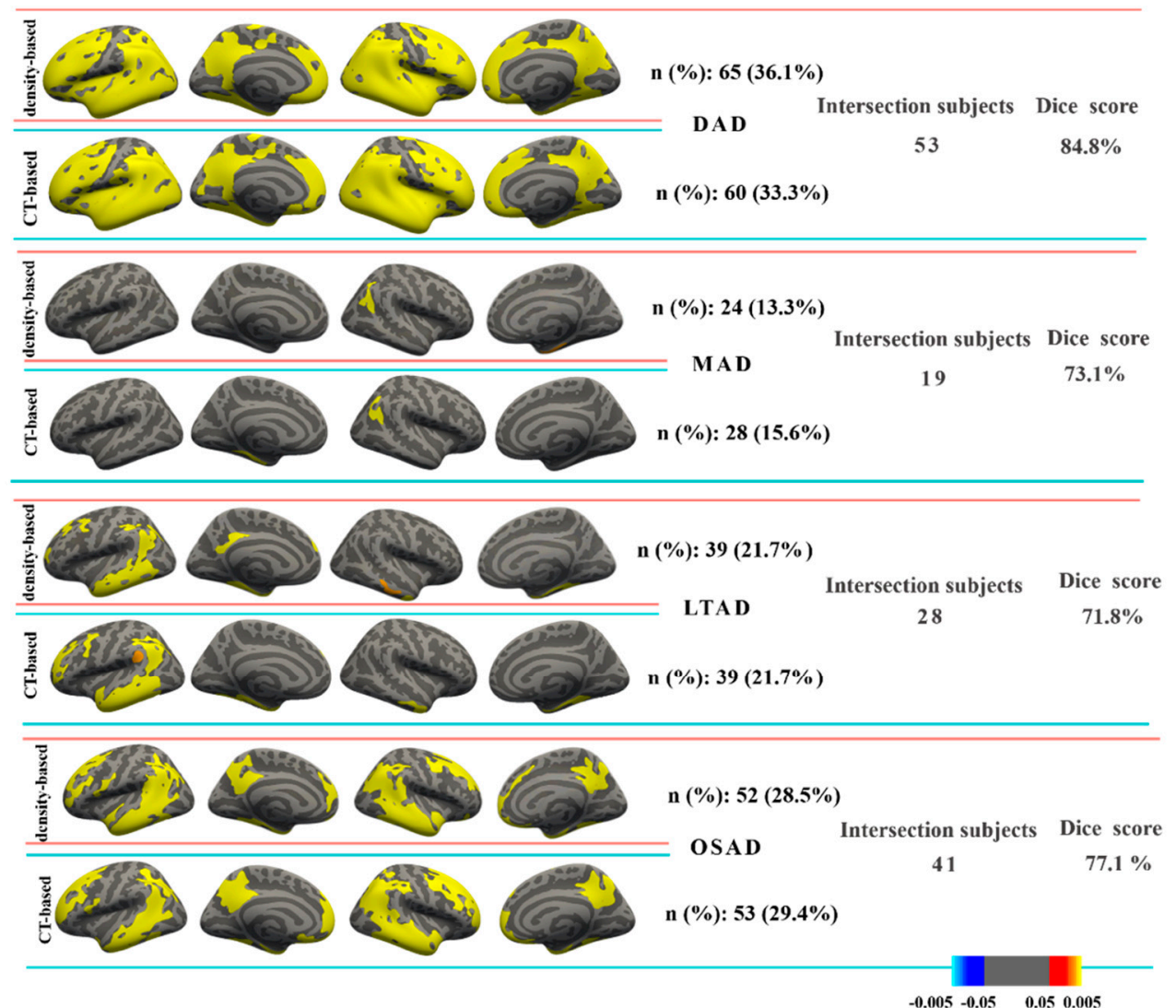

Figure 3. CT maps of subtypes defined by CT or density when compared with CNs. The brain maps were uncorrected for multiple comparisons at $p<0.05$. The statistical parametric maps included all the subjects in each subtype. Abbreviations: DAD: diffuse atrophy AD subtype, MAD: minimal atrophy AD subtype, LTAD: left temporal dominant atrophy AD subtype, OSAD: occipital sparing AD subtype, CT: cortical thickness, n: the number of AD subjects, \%: proportion of AD subjects in a subtype.

Table 2. A brief description of each subtype.

\begin{tabular}{ccc}
\hline Subtype & Description & $\begin{array}{c}\text { Demographic, Neuropsychological, and } \\
\text { Neuropathology Characteristics }\end{array}$ \\
\hline DAD & $\begin{array}{c}\text { Extensive cortical and } \\
\text { subcortical atrophy. }\end{array}$ & $\begin{array}{c}\text { Severe and extensive deficits in all cognitive } \\
\text { domains. Higher proportions of APOE } \varepsilon 4 \text { carriers, } \\
\text { higher levels of abnormal A } \beta_{1-42} \text { and P-tau. }\end{array}$ \\
\hline MAD & $\begin{array}{c}\text { With the least extent and } \\
\text { amount of atrophy in cortical } \\
\text { regions, but with sporadic } \\
\text { atrophy in subcortical regions. }\end{array}$ & $\begin{array}{c}\text { Good cognitive performance in all fields among the } \\
\text { four subtypes. With higher APOE } \varepsilon 2 \text { carriers, and } \\
\text { the lowest levels of abnormal A } \beta_{1-42}, \text { P-tau, and } \\
\text { T-tau. }\end{array}$ \\
\hline
\end{tabular}


Table 2. Cont.

\begin{tabular}{ccc}
\hline Subtype & Description & $\begin{array}{c}\text { Demographic, Neuropsychological, and } \\
\text { Neuropathology Characteristics }\end{array}$ \\
\hline LTAD & $\begin{array}{c}\text { Asymmetrical atrophy in the } \\
\text { left temporal-parietal cortex. }\end{array}$ & $\begin{array}{c}\text { A relatively low proportion of women, and a higher } \\
\text { proportion of APOE } \varepsilon 2 \text { carriers. }\end{array}$ \\
\hline OSAD & $\begin{array}{c}\text { Prominent atrophy in most of } \\
\text { cortex and subcortex, except } \\
\text { the occipital area. }\end{array}$ & The higher levels of abnormal A $\beta_{1-42}$ and T-tau. \\
\hline
\end{tabular}

Abbreviations: DAD: diffuse atrophy AD subtype, MAD: minimal atrophy AD subtype, LTAD: left temporal dominant atrophy AD subtype, OSAD: occipital sparing AD subtype. APOE: apolipoprotein, $\mathrm{A} \beta_{1-42}$ : beta-amyloid 1-42, P-tau: Phosphorylated tau, T-tau: Total tau.

\subsection{GM Density Map between Matched Subtypes}

Figure 4 presents the statistical parametric maps between AD subtype and $\mathrm{CN}$ of matched subtypes. A false discovery rate (FDR)-corrected p-value threshold of 0.05 was used. The regional GM atrophy between matched subtypes were roughly consistent. The DAD exhibited extensive atrophy. In LTAD, the GM atrophy was found in the temporal lobe ROIs, such as the hippocampus, amygdala, and fusiform. The more significant difference was manifested in the left lateralized regions. The OSAD displayed the atrophy ROIs in temporal, frontal, parietal, and posterior fossa structures. Compared to the DAD, the differences between OSAD and NC were slightly smaller.
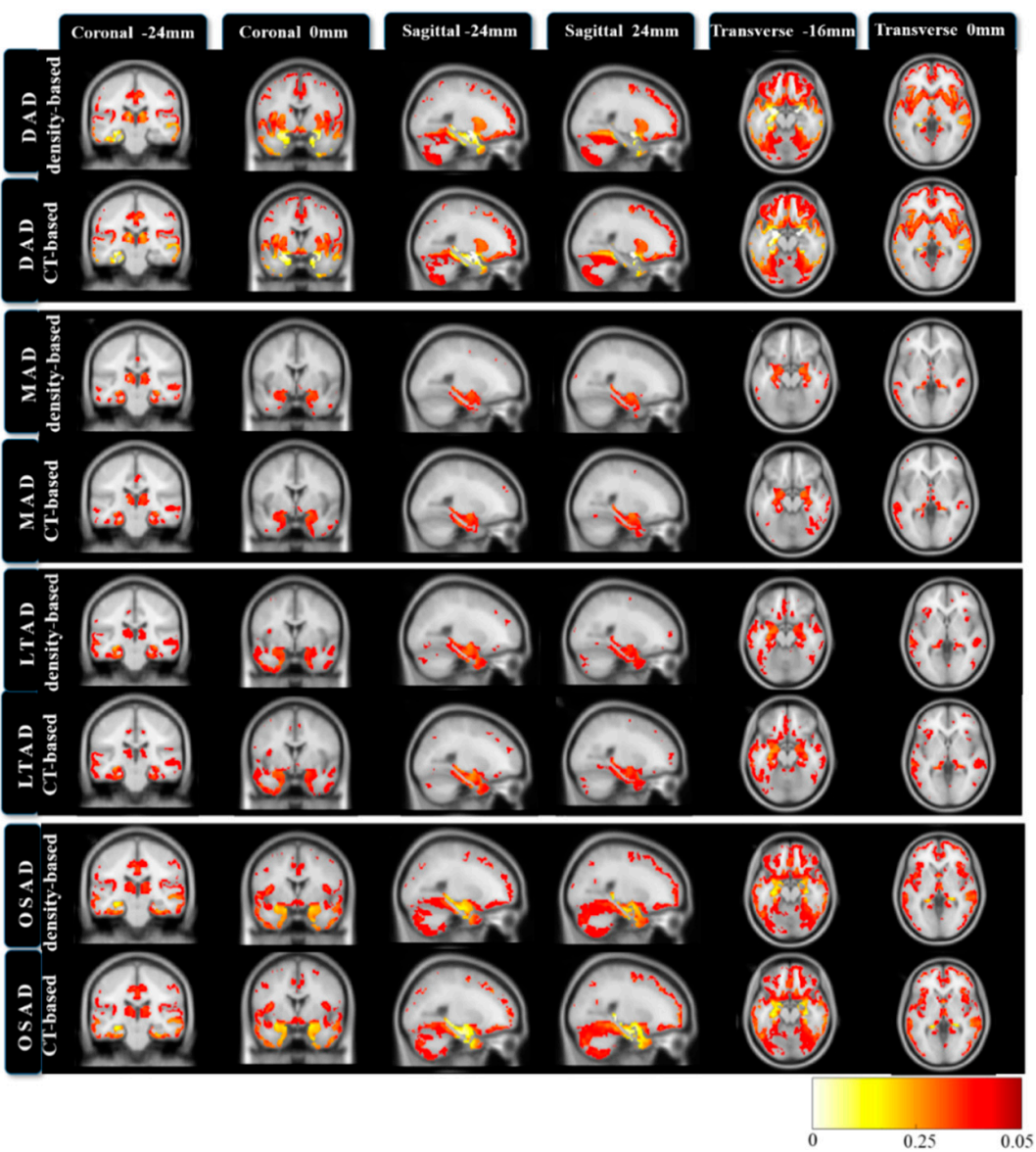

Figure 4. Statistical parametric maps of subtypes were identified by CT-based and density-based compared with the CNs. The results were thresholded at FDR corrected $p<0.05$. The statistical parametric maps included all the subjects in each subtype. Abbreviations: DAD: diffuse atrophy AD subtype, MAD: minimal atrophy AD subtype, LTAD: left temporal dominant atrophy AD subtype, OSAD: occipital sparing AD subtype, CT: cortical thickness. 


\subsection{Cognitive and Neuropathological Characteristics between Matched Subtypes}

The characteristics of atrophic regions, demographic, cognitive, APOE genotype (APOE $\varepsilon 2$ and APOE $\varepsilon 4$ ), and CSF biomarker levels are described in Figures 5 and 6 . And the characteristics of each matched subtype were summarized in Table 2. For each matched subtype, most characteristics of density-based subtypes were consistent with those of CT-based ones. The only difference was reflected in APOE $\varepsilon 2$. More APOE $\varepsilon 2$ carriers in the MAD_CT than the MAD_density, while more APOE $\varepsilon 2$ carriers were observed in LTAD_density than the LTAD_CT.

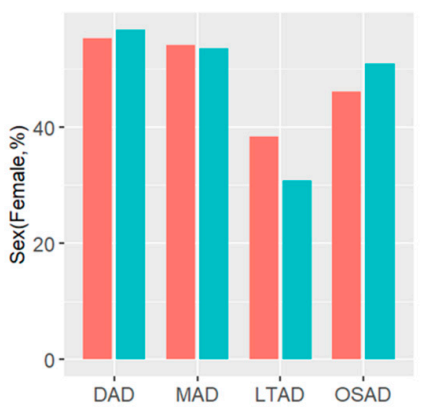

A

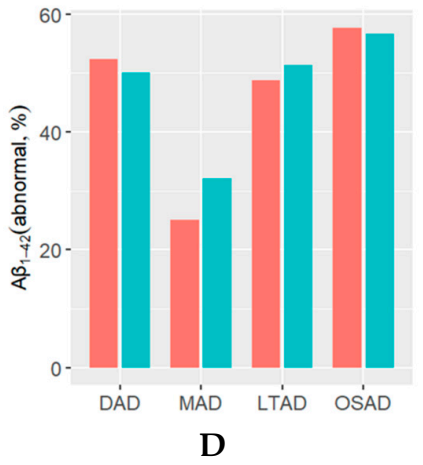

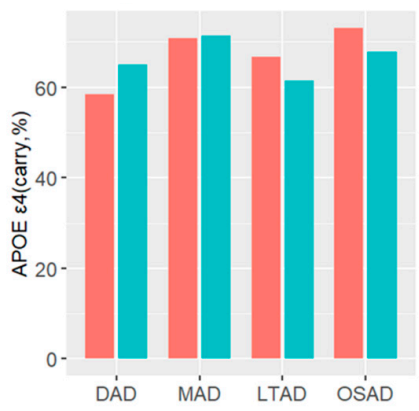

B

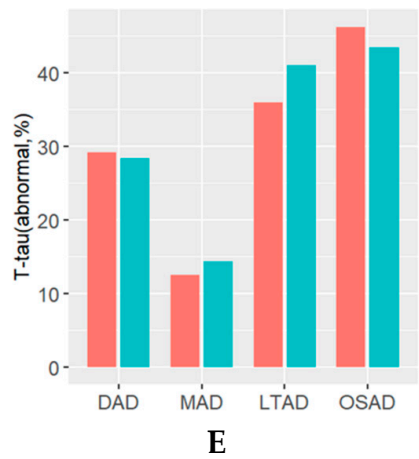

density-based CT-based

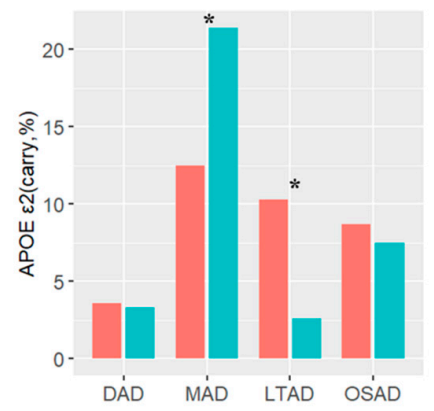

$\mathrm{C}$

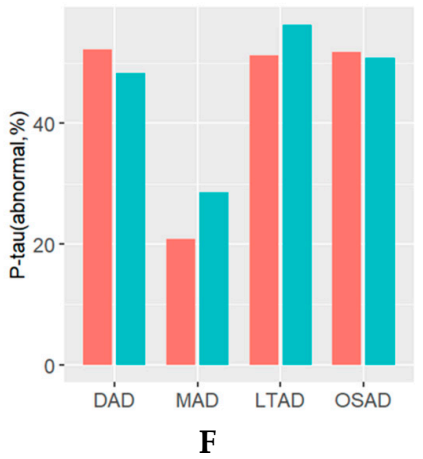

Figure 5. The characteristics of qualitative variables were captured by density features and CT features. The chi-square test was used for comparison between matched subtypes. ${ }^{*}: p<0.05$ within the matched subtype. (A-C) The histograms for sex and APOE demonstrate what percentage of AD subjects in each subtype were females and APOE carriers, respectively. (D-F) The histograms for CSF (A $\beta_{1-42}, \mathrm{~T}-\mathrm{tau}$, and P-tau) indicate the abnormal percentage of AD subjects in each subtype. Abbreviations: AD: Alzheimer's disease, APOE: apolipoprotein, $\mathrm{A} \beta_{1-42}$ : beta-amyloid 1-42, P-tau: Phosphorylated tau, T-tau: Total tau. DAD: diffuse atrophy AD subtype, MAD: minimal atrophy AD subtype, LTAD: left temporal dominant atrophy AD subtype, MAD: minimal atrophy AD subtype, OSAD: occipital sparing AD subtype. 

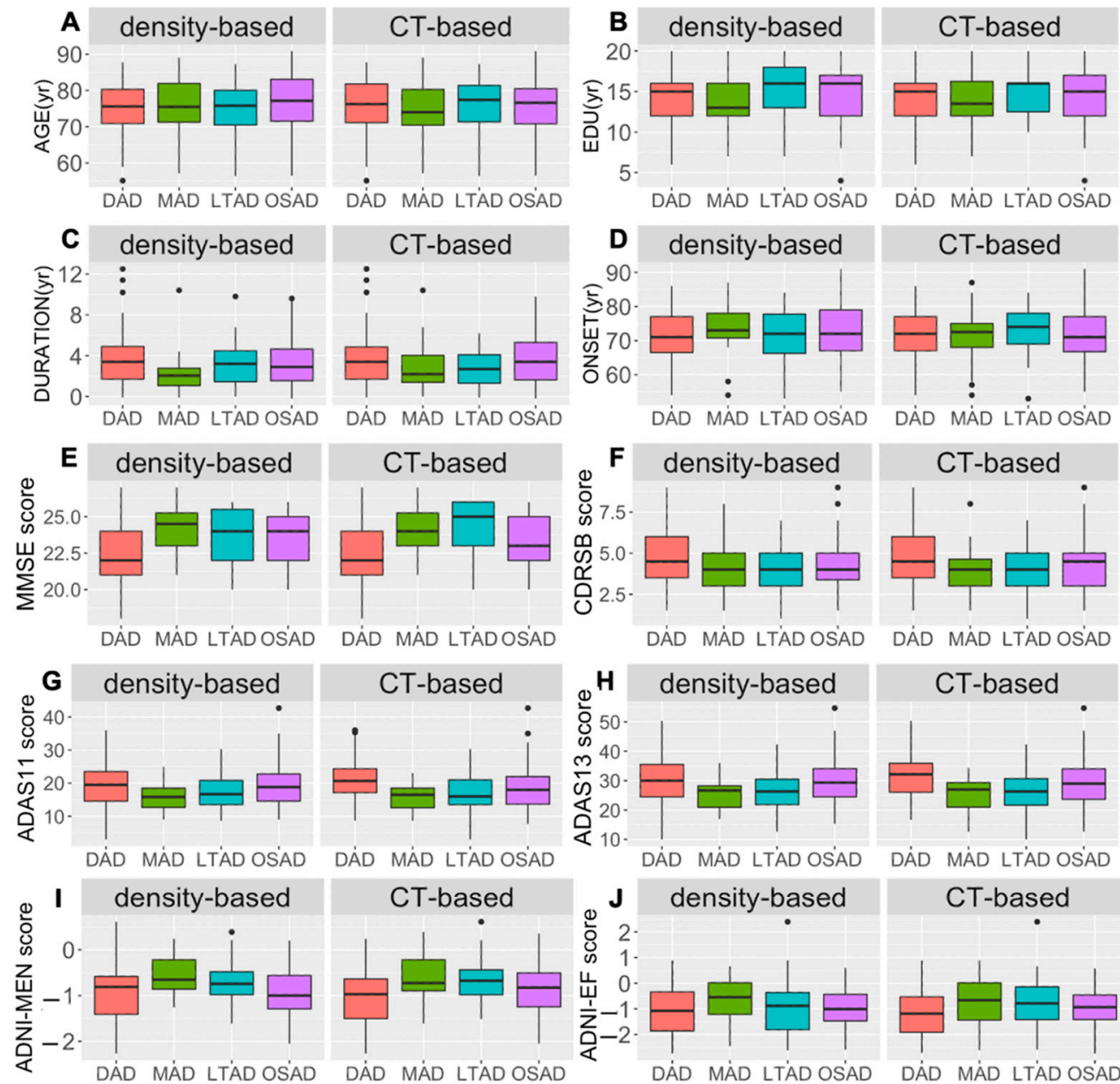

CT-based
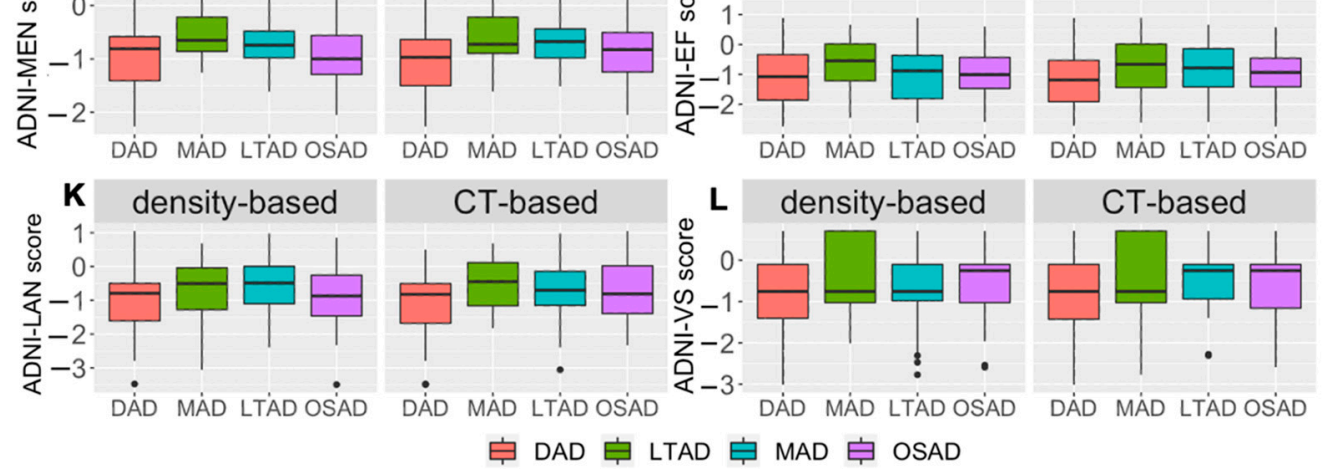

追 DAD 追 LTAD 追 MAD 追 OSAD

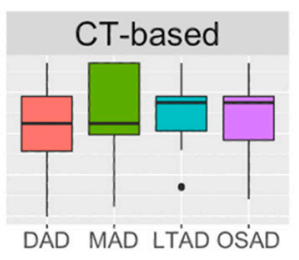

Figure 6. The characteristics of quantitative variables were captured by density features and CT features. Dunnett's test was conducted comparing the subtypes within each method. (A): Age of subjects in each subtype; (B): Years of education; (C): the duration of AD; (D): the onset age of $\mathrm{AD},(\mathbf{E}-\mathbf{H})$ : the cognitive scores of MMSE, CDR, ADAS11, and ADAS13; (I-L): the assessments of ADNI composite score. Abbreviations: EDU: education, MMSE: Mini-Mental State Exam, CDRSB: Clinical Dementia Rating Scale-Sum of Boxes, ADAS11: AD Assessment Scale-Cognitive Subscale, which includes 11 tasks, ADAS13: AD Assessment Scale-Cognitive Subscale, which includes 13 tasks, ADNI-MEM: ADNI composite scores for memory, ADNI-EF: ADNI composite scores for executive function, ADNI-LAN: ADNI composite scores for language ability, ADNI-VS: ADNI composite scores for visuospatial ability, yr: year. DAD: diffuse atrophy AD subtype, MAD: minimal atrophy AD subtype, LTAD: left temporal dominant atrophy AD subtype, OSAD: occipital sparing AD subtype.

\section{Discussion}

This study mainly aimed to test the repeatability and variation of AD subtype definition between density-based and CT-based morphological measures. It transpired that the subtypes generated by both features could morphologically correspond to each other (Figures 3 and 4). There was a good overlap between matched subtypes among these 
four subtypes in the subjects, and the neuropsychological and pathological characteristics between the matched subtypes were roughly consistent.

In previous studies, neuropathology and neuroimaging have been used to explore AD subtypes. Murray et al. found that the neurofibrillary pathological process in some AD patients followed an alternative distribution based upon a large autopsy series [2]. This was the first time the hypothesis that AD had distinct subtypes from the clinical and pathological points of view had been supported. Whitwell et al. tracked the AD subtypes in vivo by investigating the atrophy patterns in structural MRI, along with regional volumetric analysis [28]. They found three AD subtypes whose atrophy regions matched the neurofibrillary pathological results. Our current study defined the AD subtypes in a univariate way, and the brain regions were treated independently without considering the inter-regional covarying relationship of GM measures. The brain regions with covarying morphological features were observed among the subjects, suggesting shared inter-individual differences in the disease progression [29-31]. Atrophy, an established biomarker for neurodegeneration, is related to tau accumulation in certain brain regions. Tau PET imaging provides valuable information regarding tau accumulation in the human brain during aging and neurodegenerative conditions [32]. As indicated in the study, the patterns of hypometabolism noted in each subtype match well with the cortical thinning regions [7]. The aggregation of $\mathrm{A} \beta_{1-42}$, a pathological hallmark of $\mathrm{AD}$, has been reported in both cognitively impaired and cognitively normal older adults, although controversies exist over their mechanisms of causing neurodegeneration [33]. The multi-modal neuroimage analysis results revealed that the regional patterns of $A \beta_{1-42}$ deposition, glucose metabolism change, and GM atrophy presented largely overlapping distributions $[34,35]$. This result accords with the conclusion drawn in a study on AD continuum through the voxel-wise approach, namely, the spatial overlap of pathology and neurodegeneration [36]. The results of our study indicate that the consistency of regional overlap in AD subtype was not markedly influenced by different GM measurement methods.

In the analysis of matched subtype, the only significant difference was presented in the APOE $\varepsilon 2$ status of MAD and LTAD. MAD is a special subtype that has attracted widespread attention in previous studies. It has the clinical symptoms of $A D$, but with no obvious changes in cortical structure compared with normal aging (Figures 3 and 4). In previous studies, mixed results regarding MAD have been presented [37]. Several studies have shown the early onset of MAD [17,38,39]; however, a survey by Byun et al. reported conflicting results [40]. Although some investigators have said that the MMSE score of MAD is the best among all subtypes, an opposite result was obtained in a study [41]. Sometimes it is claimed that the proportion of APOE $\varepsilon 4$ carriers is the highest $[17,38,39]$; however, it was the lowest, as pointed out in various independent studies [41,42]. Abnormal $A \beta_{1-42}$ carriers in MAD accounted for the smallest proportion in Ten Kate's study [17], but the opposite result has been reported in other studies [38,40]. In our current study, the proportions of APOE $\varepsilon 2$ was the most apparent difference in the matched subtype of MAD (Figure 5C). It is widely known that APOE $\varepsilon 4$ plays an important role in clearing amyloid-beta peptides, whereas the APOE $\varepsilon 2$ variant, found in approximately $5-10 \%$ of the population, is considered neuroprotective [43,44]. The young APOE $\varepsilon 2$ carriers have higher CT values compared with the other APOE genotypes [45,46]. Elderly normal APOE $\varepsilon 2$ carriers also exhibit slower rates of hippocampal atrophy [47]. MAD is a subtype with the lowest degree of atrophy, which may be ascribed to the cortical protective effect of APOE $\varepsilon 2$ in the duration of AD. Obviously, the MAD_CT (2.8 \pm 2.2 years) had a longer disease duration than MAD density ( $2.3 \pm 2.1$ years), and the number of MAD_CT subjects was larger than that MAD density subjects. In addition to the protective effect of APOE $\varepsilon 2$, the progression of NFTs cannot be ignored. The spatial distribution of NFTs pathology is associated with neuronal and synaptic dysfunction, and neuronal loss, which initially occur in the transentorhinal cortex and then progress to the hippocampus and other neighboring cortices [1]. Compared with the density features, CT features cannot reflect the measures in 
subcortical regions. It was inferred that some MAD_CT subjects might not have obvious pathologic changes in cortex, so they were assigned to the MAD subtype.

It was noticed that $79.2 \%$ of MAD_CT subjects also belonged to the MAD density, while the rest, $20.8 \%$, were assigned to LTAD density. LTAD presented prominent atrophy in the left lateral parietal, middle, and inferior temporal, with the shortest disease duration. Furthermore, it had the least atrophic degree, except for MAD. Many previous studies have reported that the left hemisphere is more susceptible to $\mathrm{AD}$, whose changes are more severe and may precede the changes in the right hemisphere by up to two years [48,49]. Compared with CT measures, the density morphological measures may be more sensitive to detecting asymmetric subcortical atrophy during the early stage of AD.

The limitations of this study included: (1) Density features could be obtained through various toolboxes, such as FSL [50], CAT 12 (http:/ / www.neuro.uni-jena.de/ cat/, accessed on 3 January 2022), etc. The density features obtained via SPM were used in our study. Whether the processing toolbox could influence the subtype definition was uncertain, which is an interesting issue worthy of further investigation. (2) A further study in a large cohort is required. Here, an ANDI- 1 cohort of subjects (around $400 \mathrm{AD}$ or CN subjects) was used to model the definition of subtypes. There were only dozens of patients in each AD subtype. Moreover, due to the absence of nearly half of the data for beta-amyloid and tau, it is difficult to analyze the matched subtypes more deeply from a pathological perspective. (3) Studies on AD continuum show that molecular pathology and neurodegeneration show different spatial relationships in the whole course of $\mathrm{AD}$, so different subtype results may be presented using mode-varying imaging methods in different stages of $\mathrm{AD}[6,36]$. In the future, the influences of different GM features on the definitions of AD subtypes can be further explored. Moreover, the influences of mode-varying imaging methods on the definitions of $\mathrm{AD}$ subtypes in different stages of $\mathrm{AD}$ can be compared.

\section{Conclusions}

Two commonly-used neuroimaging morphological measures (CT maps from FreeSurfer's surface-based calculation and GM density maps from SPM's voxel-based calculation) in AD subtype definition were used. Next, whether or not the subtypes could be identified consistently with different morphological measures was tested. The results suggest that the two measures can identify relatively consistent subtypes. Because the patterns of atrophy in the matched group appear somewhat consistent, there are likely covarying effects between density and CT measures. For MAD and LTAD, there exists some inconsistency, which may be partially explained through APOE genotype, asymmetric disease progression, and their interactions. However, the definitive resolution will need to await the availability of a larger cohort. The results of our study can be considered as an appropriate reference for facilitating $\mathrm{AD}$ subtype definition.

Author Contributions: Conceptualization, B.Z., L.L. (Lan Lin) and S.W.; methodology, L.L. (Lan Lin) and B.Z.; validation, B.Z. and L.L. (Lan Lin); formal analysis, B.Z., X.S. and L.L. (Lingyu Liu); writing—original draft preparation, B.Z.; writing-review and editing, L.L. (Lan Lin) and S.W.; visualization, B.Z. and L.L. (Lingyu Liu); supervision, L.L. (Lan Lin) and S.W.; project administration, S.W.; funding acquisition, L.L. (Lan Lin). All authors have read and agreed to the published version of the manuscript.

Funding: This research was funded by grants from the National Natural Science Foundation of China, grant number 81971683, the Natural Science Foundation of Beijing Municipality, grant number L182010, and the Scientific Research General Project of Beijing Municipal Education Committee, grant number KM201810005033.

Institutional Review Board Statement: Not applicable.

Informed Consent Statement: Not applicable.

Data Availability Statement: Not applicable. 
Acknowledgments: The authors would like to thank the anonymous reviewers for their valuable comments and suggestions. Data used in preparation of this article were obtained from the Alzheimer's disease Neuroimaging Initiative (ADNI) database (adni.loni.usc.edu). As such, the investigators within the ADNI contributed to the design and implementation of ADNI and/or provided data but did not participate in the analysis or writing of this report. A complete listing of ADNI investigators can be found at: http://adni.loni.usc.edu/wpcontent/uploads/how_to_apply/ADNI_ Acknowledgement_List.pdf access on 3 January 2022.

Conflicts of Interest: The authors have no competing interests to declare.

\section{Abbreviations}

\begin{tabular}{|c|c|c|c|}
\hline AAL & Automated anatomical atlas & LAN & Language ability \\
\hline $\mathrm{ACC}$ & Cross-validated accuracy & LTAD & $\begin{array}{l}\text { left temporal dominant atrophy } \\
\text { AD subtype }\end{array}$ \\
\hline $\mathrm{AD}$ & Alzheimer's disease & MAD & Minimal atrophy AD subtype \\
\hline ADAS-Cog & $\begin{array}{l}\text { AD assessment scale-cognitive } \\
\text { subscale }\end{array}$ & MEM & Memory ability \\
\hline ADNI & AD Neuroimaging Initiative & MMSE & Mini-Mental State Examination \\
\hline ANOVA & Analysis of variance & $\mathrm{MOE}$ & Mixture of experts \\
\hline APOE & Apolipoprotein E & MRI & Magnetic resonance imaging \\
\hline$A \beta_{1-42}$ & Beta-amyloid 1-42 & MTL & Medial temporal lobe \\
\hline $\mathrm{BPC}$ & Bezdek partition coefficient & NFTs & Neurofibrillary tangles \\
\hline CDRSB & $\begin{array}{l}\text { Clinical dementia rating scale-sum } \\
\text { of boxes }\end{array}$ & OSAD & Occipital sparing AD subtype \\
\hline $\mathrm{CN}$ & Cognitive normal subjects & P-tau & Phosphorylated tau \\
\hline CT & Cortical thickness & ROI & Region-of-interest \\
\hline CSF & Cerebrospinal fluid & SPM & Statistical parametric mapping \\
\hline DAD & Diffuse atrophy AD subtype & SVM & Support vector machines \\
\hline $\mathrm{EF}$ & Executive function & T-tau & Total tau \\
\hline FCM & Fuzzy C-Means & VBM & Voxel-based morphometry \\
\hline GM & Gray matter & VS & Visuospatial ability \\
\hline ICV & Intracranial volume & $\mathrm{W}_{\mathrm{r}}$ & Maximum pair-wise inner- \\
\hline
\end{tabular}

\section{References}

1. Braak, H.; Braak, E. Neuropathological stageing of Alzheimer-related changes. Acta Neuropathol. 1991, 82, 239-259. [CrossRef] [PubMed]

2. Murray, M.E.; Graff-Radford, N.R.; Ross, O.A.; Petersen, R.C.; Duara, R.; Dickson, D.W. Neuropathologically defined subtypes of Alzheimer's disease with distinct clinical characteristics: A retrospective study. Lancet Neurol. 2011, 10, 785-796. [CrossRef]

3. Ferreira, D.; Nordberg, A.; Westman, E. Biological subtypes of Alzheimer disease. Neurology 2020, 94, 436-448. [CrossRef] [PubMed]

4. $\quad$ Pelkmans, W.; Ossenkoppele, R.; Dicks, E.; Strandberg, O.; Barkhof, F.; Tijms, B.M.; Pereira, J.B.; Hansson, O. Tau-related grey matter network breakdown across the Alzheimer's disease continuum. Alzheimer's Res. Ther. 2021, 13, 138. [CrossRef]

5. Zhang, B.; Lin, L.; Wu, S. A Review of Brain Atrophy Subtypes Definition and Analysis for Alzheimer's Disease Heterogeneity Studies. J. Alzheimer's Dis. 2021, 80, 1339-1352. [CrossRef]

6. Jellinger, K.A. Recent update on the heterogeneity of the Alzheimer's disease spectrum. J. Neural Transm. 2022, 129, 1-24. [CrossRef]

7. Hwang, J.; Kim, C.M.; Jeon, S.; Lee, J.M.; Hong, Y.J.; Roh, J.H.; Lee, J.-H.; Koh, J.-Y.; Na, D.L. Prediction of Alzheimer's disease pathophysiology based on cortical thickness patterns. Alzheimer's Dement. 2016, 2, 58-67. [CrossRef]

8. Groot, C.; Grothe, M.J.; Mukherjee, S.; Jelistratova, I.; Jansen, I.; van Loenhoud, A.C.; Risacher, S.L.; Saykin, A.J.; Mac Donald, C.L.; Mez, J.; et al. Differential patterns of gray matter volumes and associated gene expression profiles in cognitively-defined Alzheimer's disease subgroups. NeuroImage Clin. 2021, 30, 102660. [CrossRef]

9. Ashburner, J.; Friston, K.J. Voxel-Based Morphometry-The Methods. NeuroImage 2000, 11, 805-821. [CrossRef]

10. Fischl, B. FreeSurfer. NeuroImage 2012, 62, 774-781. [CrossRef]

11. Busovaca, E.; Zimmerman, M.E.; Meier, I.B.; Griffith, E.Y.; Grieve, S.M.; Korgaonkar, M.S.; Williams, L.M.; Brickman, A.M. Is the Alzheimer's disease cortical thickness signature a biological marker for memory? Br. Imaging Behav. 2016, 10, 517-523. [CrossRef] [PubMed] 
12. Desikan, R.S.; Ségonne, F.; Fischl, B.; Quinn, B.T.; Dickerson, B.C.; Blacker, D.; Buckner, R.L.; Dale, A.M.; Maguire, R.P.; Hyman, B.T.; et al. An automated labeling system for subdividing the human cerebral cortex on MRI scans into gyral based regions of interest. NeuroImage 2006, 31, 968-980. [CrossRef] [PubMed]

13. Clerx, L.; Jacobs, H.I.; Burgmans, S.; Gronenschild, E.H.; Uylings, H.B.; Echávarri, C.; Visser, P.J.; Verhey, F.R.; Aalten, P. Sensitivity of different MRI-techniques to assess gray matter atrophy patterns in Alzheimer's disease is region-specific. Curr. Alzheimer Res. 2013, 10, 940-951. [CrossRef] [PubMed]

14. Tzourio-Mazoyer, N.; Landeau, B.; Papathanassiou, D.; Crivello, F.; Etard, O.; Delcroix, N.; Mazoyer, B.; Joliot, M. Automated Anatomical Labeling of Activations in SPM Using a Macroscopic Anatomical Parcellation of the MNI MRI Single-Subject Brain. NeuroImage 2002, 15, 273-289. [CrossRef] [PubMed]

15. Sun, N.; Mormino, E.C.; Chen, J.; Sabuncu, M.R.; Yeo, B.T.T. Multi-modal latent factor exploration of atrophy, cognitive and tau heterogeneity in Alzheimer's disease. NeuroImage 2019, 201, 116043. [CrossRef] [PubMed]

16. Zhang, X.; Mormino, E.C.; Sun, N.; Sperling, R.A.; Sabuncu, M.R.; Yeo, B.T.T.; Alzheimer's Disease Neuroimaging Initiative Bayesian model reveals latent atrophy factors with dissociable cognitive trajectories in Alzheimer's disease. Proc. Natl. Acad. Sci. USA 2016, 113, E6535-E6544. [CrossRef]

17. ten Kate, M.; Dicks, E.; Visser, P.J.; van der Flier, W.M.; Teunissen, C.E.; Barkhof, F.; Scheltens, P.; Tijms, B.M.; Initiative, A.s.D.N Atrophy subtypes in prodromal Alzheimer's disease are associated with cognitive decline. Brain 2018, 141, 3443-3456. [CrossRef]

18. Kong, L.; Herold, C.J.; Zöllner, F.; Salat, D.H.; Lässer, M.M.; Schmid, L.A.; Fellhauer, I.; Thomann, P.A.; Essig, M.; Schad, L.R.; et al Comparison of grey matter volume and thickness for analysing cortical changes in chronic schizophrenia: A matter of surface area, grey/white matter intensity contrast, and curvature. Psychiatry Res. 2015, 231, 176-183. [CrossRef]

19. Zhang, B.; Lin, L.; Wu, S.; Al-Masqari, Z.H.M.A. Multiple Subtypes of Alzheimer's Disease Base on Brain Atrophy Pattern. Br. Sci. 2021, 11, 278. [CrossRef] [PubMed]

20. Petersen, R.C.; Aisen, P.S.; Beckett, L.A.; Donohue, M.C.; Gamst, A.C.; Harvey, D.J.; Jack, C.R.; Jagust, W.J.; Shaw, L.M.; Toga, A.W.; et al. Alzheimer's Disease Neuroimaging Initiative (ADNI). Clin. Charact. 2010, 74, 201-209. [CrossRef]

21. Jack, C.R., Jr.; Bernstein, M.A.; Fox, N.C.; Thompson, P.; Alexander, G.; Harvey, D.; Borowski, B.; Britson, P.J.; Whitwell, J.L.; Ward, C.; et al. The Alzheimer's Disease Neuroimaging Initiative (ADNI): MRI methods. J. Magn. Reson. Imaging 2008, 27, 685-691. [CrossRef] [PubMed]

22. Eavani, H.; Hsieh, M.K.; An, Y.; Erus, G.; Beason-Held, L.; Resnick, S.; Davatzikos, C. Capturing heterogeneous group differences using mixture-of-experts: Application to a study of aging. NeuroImage 2016, 125, 498-514. [CrossRef] [PubMed]

23. Bezdek, J.C.; Ehrlich, R.; Full, W. FCM: The fuzzy c-means clustering algorithm. Comput. Geosci. 1984, 10, 191-203. [CrossRef]

24. Crane, P.K.; Carle, A.; Gibbons, L.E.; Insel, P.; Mackin, R.S.; Gross, A.; Jones, R.N.; Mukherjee, S.; Curtis, S.M.; Harvey, D.; et al. Development and assessment of a composite score for memory in the Alzheimer's Disease Neuroimaging Initiative (ADNI). Br. Imaging Behav. 2012, 6, 502-516. [CrossRef]

25. Choi, S.E.; Mukherjee, S.; Gibbons, L.E.; Sanders, R.E.; Jones, R.N.; Tommet, D.; Mez, J.; Trittschuh, E.H.; Saykin, A.; Lamar, M.; et al. Development and validation of language and visuospatial composite scores in ADNI. Alzheimer's Dement. (N. Y.) 2020, 6, e12072. [CrossRef]

26. Shaw, L.M.; Vanderstichele, H.; Knapik-Czajka, M.; Figurski, M.; Coart, E.; Blennow, K.; Soares, H.; Simon, A.J.; Lewczuk, P.; Dean, R.A.; et al. Qualification of the analytical and clinical performance of CSF biomarker analyses in ADNI. Acta Neuropathol. 2011, 121, 597-609. [CrossRef]

27. Shaw, L.M.; Vanderstichele, H.; Knapik-Czajka, M.; Clark, C.M.; Aisen, P.S.; Petersen, R.C.; Blennow, K.; Soares, H.; Simon, A.; Lewczuk, P.; et al. Cerebrospinal fluid biomarker signature in Alzheimer's disease neuroimaging initiative subjects. Ann. Neurol. 2009, 65, 403-413. [CrossRef]

28. Whitwell, J.L.; Dickson, D.W.; Murray, M.E.; Weigand, S.D.; Tosakulwong, N.; Senjem, M.L.; Knopman, D.S.; Boeve, B.F.; Parisi, J.E.; Petersen, R.C.; et al. Neuroimaging correlates of pathologically defined subtypes of Alzheimer's disease: A case-control study. Lancet Neurol. 2012, 11, 868-877. [CrossRef]

29. Guo, X.; Chen, K.; Zhang, Y.; Wang, Y.; Yao, L. Regional covariance patterns of gray matter alterations in Alzheimer's disease and its replicability evaluation. J. Magn. Reson. Imaging 2014, 39, 143-149. [CrossRef]

30. Lin, L.; Wu, S. Applying spatial covariance modeling on cortical thickness measurement. In Proceedings of the 20125 th International Conference on BioMedical Engineering and Informatics, Chongqing, China, 16-18 October 2012; pp. $209-211$.

31. Seeley, W.W.; Crawford, R.K.; Zhou, J.; Miller, B.L.; Greicius, M.D. Neurodegenerative Diseases Target Large-Scale Human Brain Networks. Neuron 2009, 62, 42-52. [CrossRef]

32. Lois, C.; Gonzalez, I.; Johnson, K.A.; Price, J.C. PET imaging of tau protein targets: A methodology perspective. Br. Imaging Behav. 2019, 13, 333-344. [CrossRef] [PubMed]

33. Piccini, A.; Russo, C.; Gliozzi, A.; Relini, A.; Vitali, A.; Borghi, R.; Giliberto, L.; Armirotti, A.; D’Arrigo, C.; Bachi, A.; et al. Beta-amyloid is different in normal aging and in Alzheimer disease. J. Biol. Chem. 2005, 280, 34186-34192. [CrossRef]

34. Oh, H.; Habeck, C.; Madison, C.; Jagust, W. Covarying alterations in A $\beta$ deposition, glucose metabolism, and gray matter volume in cognitively normal elderly. Hum. Br. Mapp. 2014, 35, 297-308. [CrossRef] [PubMed]

35. Schroeder, C.; Park, M.T.M.; Germann, J.; Chakravarty, M.M.; Michels, L.; Kollias, S.; Kroll, S.L.; Buck, A.; Treyer, V.; Savaskan, E.; et al. Hippocampal shape alterations are associated with regional A $\beta$ load in cognitively normal elderly individuals. Eur. J. Neurosci. 2017, 45, 1241-1251. [CrossRef] [PubMed] 
36. Iaccarino, L.; La Joie, R.; Edwards, L.; Strom, A.; Schonhaut, D.R.; Ossenkoppele, R.; Pham, J.; Mellinger, T.; Janabi, M.; Baker, S.L.; et al. Spatial Relationships between Molecular Pathology and Neurodegeneration in the Alzheimer's Disease Continuum. Cereb. Cortex 2021, 31, 1-14. [CrossRef] [PubMed]

37. Lin, L.; Zhang, G.; Zhang, B.; Wu, S. Understanding the role of APOE Gene Polymorphisms in Minimal Atrophy Alzheimer's Disease by mixture of expert models. In Proceedings of the 2021 International Conference on Environmental and Engineering Management (EEM 2021), Changsha, China, 23-25 April 2021; Volume 253. [CrossRef]

38. Ferreira, D.; Shams, S.; Cavallin, L.; Viitanen, M.; Martola, J.; Granberg, T.; Shams, M.; Aspelin, P.; Kristoffersen-Wiberg, M.; Nordberg, A.; et al. The contribution of small vessel disease to subtypes of Alzheimer's disease: A study on cerebrospinal fluid and imaging biomarkers. Neurobiol. Aging 2018, 70, 18-29. [CrossRef] [PubMed]

39. Poulakis, K.; Pereira, J.B.; Mecocci, P.; Vellas, B.; Tsolaki, M.; Kłoszewska, I.; Soininen, H.; Lovestone, S.; Simmons, A.; Wahlund, L.-O.; et al. Heterogeneous patterns of brain atrophy in Alzheimer's disease. Neurobiol. Aging 2018, 65, 98-108. [CrossRef]

40. Byun, M.S.; Kim, S.E.; Park, J.; Yi, D.; Choe, Y.M.; Sohn, B.K.; Choi, H.J.; Baek, H.; Han, J.Y.; Woo, J.I.; et al. Heterogeneity of Regional Brain Atrophy Patterns Associated with Distinct Progression Rates in Alzheimer's Disease. PLoS ONE 2015, 10, e0142756. [CrossRef] [PubMed]

41. Persson, K.; Eldholm, R.S.; Barca, M.L.; Cavallin, L.; Ferreira, D.; Knapskog, A.-B.; Selbæk, G.; Brækhus, A.; Saltvedt, I.; Westman, E.; et al. MRI-assessed atrophy subtypes in Alzheimer's disease and the cognitive reserve hypothesis. PLoS ONE 2017, 12, e0186595. [CrossRef] [PubMed]

42. Dong, A.; Toledo, J.B.; Honnorat, N.; Doshi, J.; Varol, E.; Sotiras, A.; Wolk, D.; Trojanowski, J.Q.; Davatzikos, C.; Alzheimer's Disease Neuroimaging Initiative. Heterogeneity of neuroanatomical patterns in prodromal Alzheimer's disease: Links to cognition, progression and biomarkers. Brain 2016, 140, 735-747. [CrossRef] [PubMed]

43. Roe, C.M.; Ances, B.M.; Head, D.; Babulal, G.M.; Stout, S.H.; Grant, E.A.; Hassenstab, J.; Xiong, C.; Holtzman, D.M.; Benzinger, T.L.S.; et al. Incident cognitive impairment: Longitudinal changes in molecular, structural and cognitive biomarkers. Brain $\mathbf{2 0 1 8}$ 141, 3233-3248. [CrossRef] [PubMed]

44. Nikitidou, E.; Khoonsari, P.E.; Shevchenko, G.; Ingelsson, M.; Kultima, K.; Erlandsson, A. Increased Release of Apolipoprotein E in Extracellular Vesicles Following Amyloid- $\beta$ Protofibril Exposure of Neuroglial Co-Cultures. J. Alzheimer's Dis. 2017, 60, 305-321. [CrossRef] [PubMed]

45. Suri, S.; Heise, V.; Trachtenberg, A.J.; Mackay, C.E. The forgotten APOE allele: A review of the evidence and suggested mechanisms for the protective effect of APOE $\epsilon 2$. Neurosci. Biobehav. Rev. 2013, 37, 2878-2886. [CrossRef] [PubMed]

46. Shaw, P.; Lerch, J.P.; Pruessner, J.C.; Taylor, K.N.; Rose, A.B.; Greenstein, D.; Clasen, L.; Evans, A.; Rapoport, J.L.; Giedd, J.N. Cortical morphology in children and adolescents with different apolipoprotein E gene polymorphisms: An observational study. Lancet Neurol. 2007, 6, 494-500. [CrossRef]

47. Chiang, G.C.; Insel, P.S.; Tosun, D.; Schuff, N.; Truran-Sacrey, D.; Raptentsetsang, S.T.; Jack, C.R.; Aisen, P.S.; Petersen, R.C.; Weiner, M.W.; et al. Hippocampal atrophy rates and CSF biomarkers in elderly APOE2 normal subjects. Neurology 2010, 75, 1976-1981. [CrossRef]

48. Murphy, E.A.; Holland, D.; Donohue, M.; McEvoy, L.K.; Hagler, D.J., Jr.; Dale, A.M.; Brewer, J.B. Six-month atrophy in MTL structures is associated with subsequent memory decline in elderly controls. Neuroimage 2010, 53, 1310-1317. [CrossRef]

49. Donix, M.; Burggren, A.C.; Scharf, M.; Marschner, K.; Suthana, N.A.; Siddarth, P.; Krupa, A.K.; Jones, M.; Martin-Harris, L.; Ercoli, L.M.; et al. APOE associated hemispheric asymmetry of entorhinal cortical thickness in aging and Alzheimer's disease. Psychiatry Res. Neuroimaging 2013, 214, 212-220. [CrossRef]

50. Smith, S.M.; Jenkinson, M.; Woolrich, M.W.; Beckmann, C.F.; Behrens, T.E.J.; Johansen-Berg, H.; Bannister, P.R.; De Luca, M.; Drobnjak, I.; Flitney, D.E.; et al. Advances in functional and structural MR image analysis and implementation as FSL. NeuroImage 2004, 23, S208-S219. [CrossRef] 